PROCEEDINGS OF THE

AMERICAN MATHEMATICAL SOCIETY

Volume 128, Number 2, Pages 375-381

$\mathrm{S}$ 0002-9939(99)05078-9

Article electronically published on July 8, 1999

\title{
ABSTRACT BLOWING DOWN
}

\author{
MICHEL VAN DEN BERGH
}

(Communicated by Lance W. Small)

\begin{abstract}
Assume that $X$ is a surface over an algebraically closed field $k$. Let $\tilde{X}$ be obtained from $X$ by blowing up a smooth point and let $L$ be the exceptional curve. Let $\operatorname{coh}(X)$ be the category of coherent sheaves on $X$. In this note we show how to recover $\operatorname{coh}(X)$ from $\operatorname{coh}(\tilde{X})$, if we know the object $\mathcal{O}_{L}(L)$
\end{abstract}

\section{INTRODUCTION}

Assume that $X$ is a surface over an algebraically closed field $k$. Let $\tilde{X}$ be obtained from $X$ by blowing up a smooth point and let $L$ be the exceptional curve. Let $\operatorname{coh}(X)$ be the category of coherent sheaves on $X$. It is an interesting question to recover $\operatorname{coh}(X)$ from $\operatorname{coh}(\tilde{X})$ provided we know $N=\mathcal{O}_{L}(L)$.

The reason why we prefer to take $N$ as our basic object instead of $\mathcal{O}_{L}$ is the following. Let $\alpha: \tilde{X} \rightarrow X$ be the projection map. Then clearly $R \alpha_{*} N=0$. So in some sense $\operatorname{coh}(X)$ should be obtained from $\operatorname{coh}(\tilde{X})$ by dividing out $N$.

This does not quite work on the level of abelian categories, but it does work on the level of derived categories. In [3] it is shown that in an appropriate sense $D^{b}(\operatorname{coh}(X))=D^{b}(\operatorname{coh}(\tilde{X})) /[N]$. Hence to find $\operatorname{coh}(X)$ we have to put a $t$-structure [1] on $D^{b}(\operatorname{coh}(\tilde{X})) /[N]$. It is easier however to proceed slightly differently. From the fact that $R \alpha_{*} \mathcal{O}_{\tilde{X}}=\mathcal{O}_{X}$ one deduces that $R \alpha_{*} L \alpha^{*}$ is the identity on $D^{b}(\operatorname{coh}(X))$. Hence $L \alpha^{*}$ defines a full and faithful embedding of $D^{b}(\operatorname{coh}(X))$ in $D^{b}(\operatorname{coh}(\tilde{X}))$. Let $\mathcal{S}$ be its essential image. Then according to [3] we have

$$
\mathcal{S}=\left\{A \in D^{b}(\operatorname{coh}(\tilde{X})) \mid \operatorname{RHom}(A, N)=0\right\} .
$$

So we have to put a $t$-structure on $\mathcal{S}$ whose heart is $\operatorname{coh}(X)$. The solution to this problem is as follows. Define the following categories:

$$
\begin{aligned}
& \mathcal{T}=\{T \in \operatorname{coh}(\tilde{X}) \mid \operatorname{Hom}(T, N)=0\}, \\
& \mathcal{F}=\{F \in \operatorname{coh}(\tilde{X}) \mid \forall T \in \mathcal{T}: \operatorname{Hom}(T, F)=0\} .
\end{aligned}
$$

Following [5] we define a "perverse" $t$-structure on $D^{b}(\operatorname{coh}(\tilde{X}))$ by

$$
\begin{aligned}
& \mathcal{D}_{\leq 0}^{p}=\left\{B \in \mathcal{D}_{\leq 0} \mid H^{0}(B) \in \mathcal{T}\right\}, \\
& \mathcal{D}_{\geq 0}^{p}=\left\{B \in \mathcal{D}_{\geq-1} \mid H^{-1}(B) \in \mathcal{F}\right\} .
\end{aligned}
$$

Received by the editors April 8, 1998.

1991 Mathematics Subject Classification. Primary 14A20, 18E35.

Key words and phrases. Blowing down.

The author is a director of research at the NFWO. 
Then we have our main result.

Theorem 1.1. The perverse $t$-structure on $D(\operatorname{coh}(\tilde{X}))$ induces a t-structure on $\mathcal{S}$ and the heart of this $t$-stucture is precisely $\operatorname{coh}(X)$.

It doesn't seem obvious to give a version of this result which does not refer to derived categories.

Below we will give an abstract version of the construction outlined above. The reason is that exactly the same construction may be performed in the non-commutative case in order to find an inverse to the non-commutative blowing up introduced in $[6,7]$. The details of this more general case will be published elsewhere.

After this paper was finished I have been informed by Bondal that he has independently obtained a similar result (unpublished). I also wish to thank Bondal for reading a first version of this manuscript and for pointing out an error.

\section{Tilting}

In this section we outline a construction from [5]. Let $\mathcal{A}$ be an abelian category and let $(\mathcal{T}, \mathcal{F})$ be a torsion theory in $\mathcal{A}$. This is by definition a pair of full additive subcategories of $\mathcal{A}$ such that for every $T \in \mathcal{T}, F \in \mathcal{F}$ one has $\operatorname{Hom}(T, F)=0$ and furthermore for every $A$ there exists a (necessarily unique, up to ismorphism) exact sequence

$$
0 \rightarrow T \rightarrow A \rightarrow F \rightarrow 0
$$

with $T \in \mathcal{T}, F \in \mathcal{F}$.

From these conditions it easily follows that

$$
\begin{aligned}
& \mathcal{T}=\{T \in \mathcal{A} \mid \forall F \in \mathcal{F}: \operatorname{Hom}(T, F)=0\}, \\
& \mathcal{F}=\{F \in \mathcal{A} \mid \forall T \in \mathcal{T}: \operatorname{Hom}(T, F)=0\} .
\end{aligned}
$$

Thus $\mathcal{T}={ }^{\perp} \mathcal{F}$ and $\mathcal{F}=\mathcal{T}^{\perp}$, with obvious notation. Under suitable finiteness conditions (like $\mathcal{A}$ noetherian) (2.2), (2.3) imply (2.1).

Tilting allows one to construct a new abelian category $\mathcal{B}$ with the roles of $\mathcal{T}$ and $\mathcal{F}$ interchanged. Below let $\mathcal{D}$ stand for $D^{b}(\mathcal{A})$. One defines

$$
\begin{aligned}
& \mathcal{D}_{\leq 0}^{p}=\left\{B \in \mathcal{D}_{\leq 0} \mid H^{0}(B) \in \mathcal{T}\right\}, \\
& \mathcal{D}_{\geq 0}^{p}=\left\{B \in \mathcal{D}_{\geq-1} \mid H^{-1}(B) \in \mathcal{F}\right\}
\end{aligned}
$$

(" $p$ " stands for perverse). It is trivial to verify that this defines a $t$-structure on $\mathcal{D}$ (using the axioms in $[1, \S 1.3]$ ). Below we will denote the corresponding truncation functors by $\tau_{\leq 0}^{p}$ and $\tau_{\geq 0}^{p}$.

Let $\mathcal{B}=\mathcal{D}_{\leq 0}^{p} \cap \mathcal{D}_{\geq 0}^{p^{-}}$be the heart of this $t$-structure. It follows that this is an abelian category. Let $\left(\mathcal{T}^{\prime}, \mathcal{F}^{\prime}\right)$ be the essential images of $\mathcal{F}[1]$ and $\mathcal{T}$ is $\mathcal{B}$. Then it is easy to see that $\left(\mathcal{T}^{\prime}, \mathcal{F}^{\prime}\right)$ is a torsion pair in $\mathcal{B}$. We will call $\mathcal{B}$ the tilting of $\mathcal{A}$ with respect to the torsion theory $(\mathcal{T}, \mathcal{F})$.

Remark 2.1. Contrary to what one would expect, it is in general not possible to recover $\mathcal{A}$ from $\mathcal{B}$. In order for the role of $\mathcal{A}$ and $\mathcal{B}$ to be completely symmetric one needs additional conditions. See [5].

Remark 2.2. If $\mathcal{A}$ is noetherian, then this will not in general be the case for $\mathcal{B}$. Let $\mathcal{A}$ be the category of finitely generated abelian groups and let $\mathcal{T}, \mathcal{F}$ be respectively the ordinary torsion modules and torsion free modules. 
Since $\mathcal{A}$ is hereditary all objects are of the form $F \oplus T$ for $T \in \mathcal{T}$ and $F \in \mathcal{F}$. Similarly any object in $D^{b}(\mathcal{A})$ will be the sum of its homology. So every object in $\mathcal{B}$ will be of the form $F[1] \oplus T$. It is now a simple matter to verify that $\operatorname{RHom}(-, \mathbb{Z}[1])$ defines an equivalence between $\mathcal{B}$ and $\mathcal{A}^{\text {opp }}$. Since $\mathcal{A}$ is noetherian but not artinian, $\mathcal{B}$ will be artinian, but not noetherian.

\section{THE FORMALISM OF SEMI-ORTHOGONAL DECOMPOSITIONS}

The material in this section is taken from [2].

Let $\mathcal{D}$ be a triangulated category and let $\mathcal{B}, \mathcal{C}$ be two strict full triangulated subcategories of $\mathcal{D} .(\mathcal{B}, \mathcal{C})$ is said to be a semi-orthogonal pair if $\operatorname{Hom}_{\mathcal{D}}(B, C)=0$ for $B \in \mathcal{B}$ and $C \in \mathcal{C}$. Define

$$
\mathcal{B}^{\perp}=\left\{A \in \mathcal{D} \mid \forall B \in \mathcal{B}: \operatorname{Hom}_{\mathcal{D}}(B, A)=0\right\}
$$

${ }^{\perp} \mathcal{C}$ is defined similarly.

The following result is a slight variation of the statement of [2, Lemma 3.1].

Lemma 3.1. The following statements are equivalent for a semi-orthogonal pair $(\mathcal{B}, \mathcal{C})$.

1. $\mathcal{B}$ and $\mathcal{C}$ generate $\mathcal{D}$ (as triangulated category).

2. For every $A \in \mathcal{D}$ there exists a distinguished triangle $B \rightarrow A \rightarrow C$ with $B \in \mathcal{B}$ and $C \in \mathcal{C}$.

3. $\mathcal{C}=\mathcal{B}^{\perp}$ and the inclusion functor $i_{*}: \mathcal{B} \rightarrow \mathcal{D}$ has a right adjoint $i^{!}: \mathcal{D} \rightarrow \mathcal{B}$.

4. $\mathcal{B}={ }^{\perp} \mathcal{C}$ and the inclusion functor $j_{*}: \mathcal{C} \rightarrow \mathcal{D}$ has a left adjoint $j^{*}: \mathcal{D} \rightarrow \mathcal{C}$.

If one of these conditions holds, then the triangles in 2. are unique up to unique isomorphism. They are necessarily of the form

$$
i_{*} i^{!} A \rightarrow A \rightarrow j_{*} j^{*} A
$$

where the maps are obtained by adjointness from the identiy maps $i^{!} A \rightarrow i^{!} A$ and $j^{*} A \rightarrow j^{*} A$. In particular triangles as in 2. are functorial.

Remark 3.2. The notation $\left(i_{*}, i^{!}, j_{*}, j^{*}\right)$ are purely symbolic and shouldn't be interpreted as direct and inverse images. In fact in the main application below $i_{*}$ will be given by an inverse image!

If a pair $(\mathcal{B}, \mathcal{C})$ satisfies one of the conditions of the previous lemma, then we say that it is a semi-orthogonal decomposition of $\mathcal{D}$. For further reference we note the following diagram of arrows

$$
\mathcal{C} \underset{j^{*}}{\stackrel{j_{*}}{\rightarrow}} \underset{i_{*}}{\stackrel{i^{!}}{\rightarrow}} \mathcal{B}
$$

In the following lemma we give some relations between these arrows.

Lemma 3.3. One has:

$$
\begin{gathered}
i^{!} i_{*}=\operatorname{id}_{\mathcal{B}}, \\
j^{*} j_{*}=\operatorname{id}_{\mathcal{C}} \\
j^{*} i_{*}=0 \\
i^{!} j_{*}=0 .
\end{gathered}
$$


In the sequel we will slightly extend the meaning of the notion of semi-orthogonality. Assume that we have functors

$$
\mathcal{C} \stackrel{j_{*}}{\longrightarrow} \mathcal{D} \stackrel{i_{*}}{\longleftarrow} \mathcal{B}
$$

which are fully faithful. Assume furthermore that the essential images of $\mathcal{B}$ and $\mathcal{C}$ in $\mathcal{D}$ are semi-orthogonal in $\mathcal{D}$. Then, if no confusion can arise, we will also call $(\mathcal{B}, \mathcal{C})$ a semi-orthogonal pair in $\mathcal{D}$. This is similar for a semi-orthogonal decomposition.

\section{Abstract Blowing Down}

Let $k$ be a field and let $\mathcal{A}$ be a noetherian $k$-linear abelian category. As before $\mathcal{D}$ stands for $D^{b}(\mathcal{A})$. Below we sometimes need $\mathrm{RHom}(-,-)$ between objects in $D^{b}(\mathcal{A})$. In order to compute this we let $\overline{\mathcal{A}}$ be the closure of $\mathcal{A}$ under direct limits [4] and we identify $D^{b}(\mathcal{A})$ with $D_{\mathcal{A}}^{b}(\overline{\mathcal{A}})$. We then compute RHom in the latter category, which is possible since $\overline{\mathcal{A}}$ has enough injectives.

Let $N$ be an object of $\mathcal{A}$ satisfying the following properties.

1. $\operatorname{RHom}(N, N)=k$.

2. For all $A \in \mathcal{A}$ and for all $i$ one has $\operatorname{dim} \operatorname{Ext}^{i}(A, N)<\infty$.

3. $\operatorname{cd} \operatorname{Hom}(-, N) \leq 2$.

4. The functor $\operatorname{Ext}^{2}(-, N)^{*}$ is representable.

We will show in this section that it is possible to define an abstract analogue of the construction outlined in the introduction.

We define a torsion theory in $\mathcal{A}$ associated to $N$ by $\mathcal{T}=\{T \in \mathcal{A} \mid \operatorname{Hom}(T, N)=$ $0\}$ and $\mathcal{F}=\mathcal{T}^{\perp}$.

The functor $-\stackrel{L}{\otimes} N$ going from $D_{f}^{b}(k)$ to $\mathcal{D}$ has a left adjoint that is given by RHom $(-, N)^{*}$. Furthermore the appropriate composition of these functors is the identity by condition 1 . So $-\stackrel{L}{\otimes} N$ is a full faithful embedding of $D_{f}^{b}(k)$ in $\mathcal{D}$. According to Lemma 3.1.4. we can now construct a diagram as in (3.2)

$$
D_{f}^{b}(k) \underset{\operatorname{RHom}(-, N)^{*}}{\stackrel{-\stackrel{\unrhd}{\otimes} N}{\rightleftarrows}} \mathcal{D} \underset{L}{\stackrel{R}{\rightleftarrows}} \mathcal{S}
$$

where

$$
\mathcal{S}=\{A \in \mathcal{D} \mid \operatorname{RHom}(A, N)=0\} .
$$

So $L: \mathcal{S} \rightarrow \mathcal{D}$ is the inclusion functor and and $R$ is its right adjoint.

Theorem 4.1. Define

$$
\begin{aligned}
& \mathcal{S}_{\leq 0}=\mathcal{D}_{\leq 0}^{p} \cap \mathcal{S}, \\
& \mathcal{S}_{\geq 0}=\mathcal{D}_{\geq 0}^{p} \cap \mathcal{S} .
\end{aligned}
$$

Then $\left(\mathcal{S}_{\leq 0}, \mathcal{S}_{\geq 0}\right)$ is a t-structure on $\mathcal{S}$.

Proof. The only non-trivial axiom is $[1, \S 1.3($ iii)] which says that for $X \in \mathcal{S}$ there should be a triangle $(A, X, B)$ with $A \in \mathcal{S}_{\leq 0}$ and $B \in \mathcal{S}_{\geq 1}$. Now we claim that in fact $\tau_{\leq 0}^{p} X \in \mathcal{S}$ and $\tau_{\geq 1}^{p} X \in \mathcal{S}$. This clearly shows what we want.

We need some preparatory work.

Lemma 4.2. If $A \in \mathcal{F}$, then $\operatorname{Ext}^{2}(A, N)=0$. 
Proof. Let $M$ be the object representing $\operatorname{Ext}^{2}(-, N)^{*}$. Assume $\operatorname{Ext}^{2}(A, N) \neq 0$. Then

$$
0 \neq \operatorname{Ext}^{2}(A, N)=\operatorname{Hom}(M, A)^{*} .
$$

The proof is complete if we show that $M \in \mathcal{T}$. However this is clear since

$$
\operatorname{Hom}(M, N)=\operatorname{Ext}^{2}(N, N)^{*}=0 .
$$

Now apply $\operatorname{Hom}(-, N)$ to the triangle

$$
\tau_{\leq 0}^{p} X \rightarrow X \rightarrow \tau_{\geq 1}^{p} X \rightarrow
$$

This yields

$$
\operatorname{Hom}^{i}\left(\tau_{\leq 0}^{p} X, N\right)=\operatorname{Hom}^{i+1}\left(\tau_{\geq 1}^{p} X, N\right) .
$$

It is clear that

$$
\operatorname{Hom}^{i}\left(\tau_{\leq 0}^{p} X, N\right)=0 \quad \text { for } i \leq 0
$$

and by the previous lemma together with condition 3 we also have

$$
\operatorname{Hom}^{j}\left(\tau_{\geq 1}^{p} X, N\right)=0 \quad \text { for } j \geq 2 .
$$

Combining (4.1), (4.2), (4.3) yields $\operatorname{Hom}^{i}\left(\tau_{\leq 0}^{p} X, N\right)=0, \operatorname{Hom}^{j}\left(\tau_{\geq 1}^{p} X, N\right)=0$ for all $i, j$. This finishes the proof.

Let $\mathcal{C}$ be the heart of the $t$-structure on $\mathcal{S}$. We view $\mathcal{C}$ as the abstract blowing down of $N$ in $\mathcal{A}$. So objects in $\mathcal{C}$ are represented by complexes

$$
A \equiv\left(A_{-1} \stackrel{\theta}{\rightarrow} A_{0}\right)
$$

such that $H_{-1}=\operatorname{ker} \theta \in \mathcal{F}$ and $H_{0}=\operatorname{coker} \theta \in \mathcal{T}$ also satisfy $\operatorname{RHom}(A, N)=0$.

One problem we have not been able to resolve is the following.

Question 4.3. In the generality above does one necessarily have that $\mathcal{C}$ is noetherian? If not, what additional conditions are necessary?

\section{The commutative Case}

What remains to be checked is that in the commutative case our hypotheses are verified, and furthermore that we get the correct answer.

So let us return to the situation from the introduction. Assume $X$ is a surface over an algebraically closed field $k$ and $\tilde{X}$ is obtained by blowing up a smooth point $p$. We have a commutative diagram

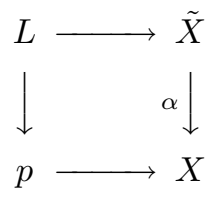

where $L$ is the exceptional curve. We put $\mathcal{A}=\operatorname{coh}(\tilde{X}), N=\mathcal{O}_{L}(L)$. Other notation will be as above.

To verify the hypotheses we note that they can all be verified in a neighborhood of $p$. Whence we may assume that $X$ is smooth. As a next step we will replace $X$ by a smooth compactification. Then condition 2 is clear and conditions 3 and 4 follow from the sophisticated version of the Serre duality

$$
\operatorname{Ext}^{i}(U, V)=\operatorname{Ext}^{2-i}\left(V, \omega_{\tilde{X}} \otimes_{\mathcal{O}_{\tilde{X}}} U\right)^{*} .
$$


Condition 1 follows easily from the long exact sequence for $\operatorname{Hom}\left(-, \mathcal{O}_{L}(L)\right)$ associated with the short exact sequence

$$
0 \rightarrow \mathcal{O}_{\tilde{X}} \rightarrow \mathcal{O}_{\tilde{X}}(L) \rightarrow \mathcal{O}_{L}(L) \rightarrow 0 .
$$

According to [3] we have a semi-orthogonal decomposition

$$
D_{f}^{b}(k) \underset{\operatorname{RHom}\left(-, \mathcal{O}_{L}(L)\right)^{*}}{\stackrel{-}{\otimes}} D^{b}(\operatorname{coh}(\widetilde{X})) \underset{L \alpha^{*}}{\stackrel{R \alpha_{*}}{\rightleftarrows}} D^{b}(\operatorname{coh}(X)) .
$$

Thus if we put

$$
\mathcal{S}=\{A \in \mathcal{D} \mid \operatorname{RHom}(A, N)=0\},
$$

it follows from Lemma 3.1 that there are inverse equivalences

$$
\mathcal{S} \underset{L \alpha^{*}}{\stackrel{R \alpha_{*}}{\rightleftarrows}} D^{b}(\operatorname{coh}(X))
$$

The canonical $t$-structure on $D^{b}(\operatorname{coh}(X))$ induces a $t$-structure on $\mathcal{S}$. We have to show that it coincides with the one we have defined earlier. That is we have to show that

$$
\begin{aligned}
& \mathcal{S}_{\leq 0}=L \alpha^{*}\left(D^{b}(\operatorname{coh}(X))_{\leq 0}\right), \\
& \mathcal{S}_{\geq 0}=L \alpha^{*}\left(D^{b}(\operatorname{coh}(X))_{\geq 0}\right) .
\end{aligned}
$$

Since $\mathcal{S}_{\geq 0}=\mathcal{S}_{\leq-1}^{\perp}$, and similarly for $D^{b}(\operatorname{coh}(X))_{\geq 0}$ it suffices to verify the first of these equalities.

Let us first show "ᄃ". Since $\mathcal{S}_{\leq 0} \subset \mathcal{S}$ and $L \alpha^{*} R \alpha_{*}$ is the identity on $\mathcal{S}$ it suffices to show that $R \alpha_{*} \mathcal{S}_{\leq 0} \subset D^{b}(\operatorname{coh}(X))_{\leq 0}$. To this end it suffices to show that if $A \in \mathcal{T}$, then $R^{1} \alpha_{*} A=0$.

The triangle

$$
L \alpha^{*} R \alpha_{*} A \rightarrow A \rightarrow \operatorname{RHom}(A, N)^{*} \otimes N \rightarrow
$$

obtained from (3.1) yields $H^{1}\left(L \alpha^{*} R \alpha_{*} A\right)=0$. Now $H^{1}\left(L \alpha^{*} R \alpha_{*} A\right)=\alpha^{*} R^{1} \alpha_{*} A$. If $R^{1} \alpha_{*} A \neq 0$, then also $\alpha^{*} R^{1} \alpha_{*} A \neq 0$ (since $\alpha$ is surjective) and we obtain a contradiction.

Now we prove the opposite inclusion. Let $T \in D^{b}(\operatorname{coh}(X))_{\leq 0}$. Clearly $L \alpha^{*} T \in$ $\mathcal{S} \cap \mathcal{D}_{\leq 0}$. Furthermore

$$
\operatorname{Hom}\left(H^{0}\left(L \alpha^{*} T\right), N\right)=\operatorname{Hom}\left(L \alpha^{*} T, N\right)=\operatorname{Hom}\left(T, R \alpha_{*} N\right)=0
$$

whence $H^{0}\left(L \alpha^{*} T\right) \in \mathcal{T}$. This proves what we want.

\section{REFERENCES}

[1] A. Beilinson, J. Bernstein, and P. Deligne, Faisceaux pervers, Astérisque, vol. 100, Soc. Math. France, 1983. MR 86g:32015

[2] A. I. Bondal, Representations of associative algebras and coherent sheaves, Math. USSR-Izv. 34 (1990), no. 1, 23-42. MR 90i:14017

[3] A. I. Bondal and D. O. Orlov, Semi-orthogonal decompositions for algebraic varieties, MPI preprint, 1996.

[4] P. Gabriel, Des catégories abéliennes, Bull. Soc. Math. France 90 (1962), 323-448. MR 38:1144 
[5] D. Happel, I. Reiten, and S. Smalø, Tilting in abelian categories and quasitilted algebras, Memoirs of the AMS, vol. 575, Amer. Math. Soc., 1996. MR 97j:16009

[6] M. Van den Bergh, Blowing up of non-commutative smooth surfaces, to appear, 1997.

[7] _ Blowing up points in the elliptic quantum plane, in preparation, 1998.

Departement WNi, Limburgs Universitair Centrum, Universitaire Campus, 3590 Diepenbeek, Belgium

E-mail address: vdbergh@luc.ac.be

$U R L:$ http://www.luc.ac.be/Research/Algebra

Current address: Department of Mathematics, Massachusetts Institute of Technology, 77 Massachusetts Avenue, Cambridge, Massachusetts 02139 